\title{
Arborização do Bairro Peixe-gordo na cidade de Icapuí-Ce
}

\author{
Afforestation neighborhood fish fat in the city of Icapui-Ce
}

Danilo Isac Maia de Souza ${ }^{1 *}$, Lucas Ramos da Costa ${ }^{2}$, Marcelo Tavares Gurgel ${ }^{3}$ Sílvio Roberto Fernandes Soares

Resumo: Este trabalho foi desenvolvido com o objetivo de avaliar a arborização do bairro de peixe-gordo. Para isso foi realizado levantando, identificação e caracterização das árvores plantadas com potencial para o uso em arborização urbana, de forma a oferecer novas opções de utilização para estas árvores, levando em conta suas qualidades e limitações. A presente pesquisa foi realizada entre os dias 13 à 28 de julho de 2013, os dados foram coletados através de contagem simples e anotados em uma ficha de campo previamente preparada com informações sobre a data de coleta, nome vulgar (popular) e científico das espécies. Ainda foi realizado o registro de fotos das árvores. Foram encontrados 266 indivíduos arbóreos, pertencente a 29 espécies. A espécie mais frequente constatada foi o coqueiro (Cocos nucifera L.) Com uma percentagem de 31,2\%. Vale ressaltar que, a presença das espécies do (a): Nim (Azadirachta indica a. Juss.) com percentual de 26,3\%, carnaúba (Copernicia prunifera) com 6,01\%, mangueira (Mangifera indica L.) e tamarindo (Tamarindus indica L.) com 4,13\% respectivamente, e o juazeiro (Ziziphus joazeiro Mart.) com 3,38\% de ocorrência.

Palavras-chave: paisagismo, plantas nativas, educação ambiental, meio ambiente

\begin{abstract}
This work was carried out to evaluate the afforestation of the district-fat fish. To this was accomplished raising, identification and characterization of planted trees with potential for use in urban forestry, in order to offer new options to use these trees, taking into account their strengths and limitations. This survey was conducted between 13th to July 28th 2013, data were collected through a simple counting and recorded on a field form previously prepared with information about the date of collection, common name (popular) and scientific species . Although the record was made of photos of trees. Found 266 individual trees belonging to 29 species. The species most frequently found was the coconut (Cocos nucifera L.) With a share of $31.2 \%$. It is noteworthy that the presence of the species (a): Neem (Azadirachta indicates. Juss.)With a percentageof $26.3 \%$, carnauba (Copernicia prunifera) with $6.01 \%$, mango (Mangifera indica L.) e tamarind (Tamarindus indica L.)With $4.13 \%$ respectively, and the jujube (Ziziphus joazeiro Mart.) With $3.38 \%$ occurrence.
\end{abstract}

Keywords: landscaping, native plants, environmental education, environment

\section{INTRODUÇÃO:}

Os solos do nordeste brasileiro são férteis e podem se tornar produtivos, desde que manejados adequadamente para solucionar principalmente seus problemas químicos e permitir maior capacitação e retenção de água. Os solos das regiões áridas e semiáridas, pelos altos teores de sais solúveis que contém nos horizontes superficiais, são salinos e alcalinos ou sódicos (halomórficos) devido às altas taxas de evapotranspiração e à baixa pluviosidade. A zona costeira do Ceará tem condições extremamente atrativas e privilegiadas para os mais variados assentamentos humanos. No entanto, a fragilidade ambiental e a vulnerabilidade ao antropismo justificam os cuidados legais para a sua preservação ou conservação, através de preceitos que se fundamentam no desenvolvimento sustentável. (SEMACE,2005).

Sua configuração física, de forma linear, permeia todas as comunidades praianas com pequena distância entre as comunidades. Percebe-se que esta forma de ocupação se relaciona com o tipo de exploração econômica realizado em períodos passados, onde as áreas diretamente anteriores às praias eram utilizadas para agropecuária, além do facilitado acesso ao mar, promovendo seu crescimento nesta porção, o município também se desenvolve ao longo da estrada que dá acesso as demais localidades.

Segundo os moradores locais entrevistados durante trabalho de campo, o adensamento aumentou depois que a estrada translitorânea foi concluída, estando atualmente toda asfaltada inclusive no trajeto para algumas

*autor para correspondência

${ }^{1}$ Graduando Em Engenharia Agronômica-UFERSA, Universidade Federal rural do Semi-árido, Av. Francisco Mota, n. 572, Costa e Silva, caixa postal 137 59625-900, Mossoró-RN. E-mail: Danilo.Isac@gmail.com

${ }^{2}$ Eng. Agrônomo, Mestrando em Manejo de Solo e Água - UFERSA, Universidade Federal rural do Semi-árido, Av. Francisco Mota, n. 572, Costa e Silva, caixa postal 137 59625-900, Mossoró-RN. E-mail: lucas_ramosjp@ @otmail.com

${ }^{3}$ Professor Dr. Sc. Adjunto III do Departamento De Ciências Ambientais e Tecnológicas Da UFERSA,Universidade Federal rural do Semi-árido, Av. Francisco Mota, n. 572 , Costa e Silva, caixa postal 137 59625-900, Mossoró-RN. E-mail: Marcelo.Tavares@Ufersa.Edu.Br 
comunidades. O processo de urbanização da cidade, a exemplo do que é experimentado pela maioria dos municípios do Ceará, segue um padrão típico caracterizado pelo desenvolvimento de relações econômicas de pequeno porte, parte delas ainda vinculadas às atividades típicas das áreas rurais.

O que é mais marcante nesta região é a possível conservação dos seus recursos, dentro do objetivo do desenvolvimento sustentável, visto seu relevante nível de associativismo, tendo como um dos frutos a conquista das áreas de preservação sob seus domínios. Apesar desta evidente conservação de parte de seus recursos naturais, algumas atividades antrópicas (causadas pela ação do homem) já resultaram na perda de qualidade ambiental.

Um dos problemas é originário, a exemplo de vários municípios litorâneos, das fazendas de criação de camarão, que vem sendo responsabilizadas por problemas da qualidade dos ambientes hídricos e a escassez de água para consumo da comunidade, além da retirada de vegetação dos manguezais e aterramento de áreas alagadas.

Também a atividade salineira causou e ainda vem causando a redução nas áreas dos manguezais e das várzeas no município de Icapuí, necessitando, contudo, de estudos e pesquisas que apontem o total de áreas degradadas e quais as medidas a serem tomadas para sua recuperação. Problemas que afetam a zona litorânea do Ceará são detectáveis de modo notável. Alguns são comuns a toda a zona costeira, como: migração de dunas, erosão da linha da costa, desmatamentos de manguezais e de matas ciliares, assoreamento ou aterramento de lagoas ou de áreas de acumulação inundáveis, poluição dos recursos hídricos superficiais, deposição de resíduos sólidos, entre outros, são alguns problemas que afetam e impactam a zona costeira. (SOUZA, 2003).

Mas um dos impactos mais graves que vem comprometendo a qualidade ambiental da paisagem do Ceará, sendo verificada também em Icapuí, são as intervenções antrópicas não planejadas como, por exemplo, a retirada indiscriminada das escarpas para a construção civil e vias de acesso (MEIRELES, 1999). Na economia de Icapuí predomina o setor primário sendo direcionado para a pesca, a extração do sal, as culturas do caju e do coco e a extração do petróleo.

A cada dia, a cidade vem ganhando mais destaque e interesse na vida de cada indivíduo, uma vez que, passo a passo, a humanidade caminha para uma vida eminentemente urbana, definida em seus aspectos quantitativos e qualitativos, suas dinâmicas e conteúdos. Ela reproduz a história, assim como as relações que o homem teve, e tem do espaço, do habitar, do trabalhar, do comer, do beber, do conviver, enfim do viver (BONAMETTI, 2000).

É de suma importância discutir e analisar o papel da arborização urbana para um melhor aproveitamento dos espaços não-edificados da cidade, melhorando assim a qualidade de meio ambiente. Para tanto, se faz indispensável à efetivação da política de pesquisa e estudo de como a arborização das vias urbanas pode preencher corretamente o espaço da cidade, para depois se proporem medidas adequadas à melhoria funcional e estética destas áreas. A questão da arborização urbana é sempre o reflexo da relação entre o homem e a natureza, e pode ser vista como uma tentativa de ordenar o ambiente com base em uma paisagem natural.

A vegetação nativa desempenha importantes funções para a preservação dos ecossistemas, como a proteção do solo, o controle sobre o regime de chuvas e do fluxo das águas, o controle da poluição atmosférica e a sobrevivência da fauna. Além disso, fornece matériaprima para diferentes finalidades (extração de lenha, estacas...) e podem ser utilizadas para atividades de lazer.

\section{MATERIAL E MÉTODOS}

A pesquisa foi desenvolvida no bairro peixe-gordo na cidade de Icapuí-CE, que se encontra a uma distância de $195 \mathrm{~km}$ da capital fortaleza, via rodoviária, fazendo parte da bacia do rio Jaguaribe, localizado no litoral leste do estado do Ceará, na mesorregião do Jaguaribe, limita-se ao norte com o oceano atlântico, ao sul e a oeste com o município de Aracati, ao leste com o estado do rio grande do norte, cuja distância para a capital, natal é de $350 \mathrm{~km}$. (PETROBRAS 2005).

Icapuí possui 18.746 habitantes e densidade demográfica de 37,41 habitantes/ $/ \mathrm{km}^{2}$ (IBGE, 2012). Deste contingente, 13.736 concentram-se na área rural, ou seja, quase $71 \%$ do total e 5.010 na área urbana (IBGE, 2012). Grande parte da sua população, maioria pescadores, vive em aglomerados em pequenos núcleos praieiros. A taxa de crescimento anual é de $2,14 \%$, percebe-se que a tendência de crescimento do município obedeceu ao mesmo quadro do ano de 1991, mas reduziu significativamente sua taxa de urbanização, já que a taxa média de crescimento anual de Icapuí passou de 37,33 \% para 29,04\% (uma redução considerável).(PETROBRAS 2005).

O município está dividido em três distritos: Icapuí (sede), Ibicuitaba e Manibú. Possui quatro bairros, Mutamba, Cajuais, Salgadinho II e Olho d'água, com trinta núcleos populacionais, localizados nas quatorze (14) praias existentes, bem como à margem da rodovia CE-261 no entroncamento da BR-304 que liga o Ceará ao rio grande do norte.

A maioria das suas comunidades é considerada "vila de pescadores" como as de Retiro Grande, Ponta Grossa, Redonda, Peróba, Picos, Barreiras, Requenguela, Quitérias, Tremenbé, Melancias e Peixe-Gordo.

O solo é composto de areias quartzosas distróficas (acúmulo de grande quantidade de matéria orgânica), com alto teor de salinidade, direcionado para a cultura de subsistência, fruticultura (coco e caju) e a pecuária extensiva. A geomorfologia é de grande valor paisagístico, composta basicamente por planície litorânea, dunas, rios e lagos. O município dispõe de cerca de $64 \mathrm{~km}$ de praias e de $40 \mathrm{~km}^{2}$ de área verde, coqueirais, manguezais, dunas, falésias e lagoas, atrativos naturais básicos para o investimento em turismo ecológico, que já vem despontando em toda a região.(PETROBRAS 2005).

Atualmente a base da agricultura é de pequeno porte, mas, por outro lado, se caracteriza por apresentar condições naturais para o desenvolvimento de certas culturas, entre elas a do caju vindo, em seguida, o coco, a mandioca, o milho e o feijão. Estas três últimas são culturas de subsistência plantadas mais para o consumo familiar do que para a comercialização. 
A presente pesquisa foi realizada entre os dias 13 à 28 de julho de 2013, os dados foram coletados através de contagem simples e anotados em uma ficha de campo previamente preparada com informações sobre a data de coleta, nome vulgar (popular) e científico. Ainda foi feito o registro de fotos das árvores. Sendo assim com base nos dados apresentados, pode-se avaliar a arborização do bairro peixe-gordo em Icapuí-ce.

\section{RESULTADO E DISCUSSÃO}

Foram encontrados 266 indivíduos arbóreos, pertencente a 29 espécies e ao mesmo tempo, calculados o numero de ocorrências e frequência (tabela 1). A espécie mais Frequente constatada foi o coqueiro (Cocos nucifera L.) Com percentagem de 31,2\%. Isso pode ser Justificado pelo fato da região de Icapuí ser tipicamente de vegetação litorânea, sendo que é a palmeira de maior importância econômica e de mais ampla distribuição geográfica.

Merecem destaque também, as espécies do (a): Nim (Azadirachta indica a. Juss.) Com percentual de 26,3\%, carnaúba (Copernicia prunifera) com 6,01\%, mangueira (Mangifera indica L.) E tamarindo (Tamarindus indica L.) Com $4,13 \%$ respectivamente, e o juazeiro (Ziziphus joazeiro Mart.) Com 3,38\% de ocorrência. (tabela 1).

tabela 1. Demonstração do número de ocorrências e frequência das plantas encontradas no bairro Peixe-Gordo na cidade de Icapuí $\mathrm{CE}$ em julho de 2013 (*E=exóticas, **N=nativas)

\begin{tabular}{|c|c|c|c|c|c|}
\hline ESPÉCIE & NOME CIENTIFICO & $N^{\circ}$ DE OCORRÉNCIAS & FREQUĖNCIA (\%) & $E^{*}$ & $\mathrm{~N}^{* *}$ \\
\hline ALGAROBEIRA & PROSOPIS JULIFRORA (SW)DC. & 2 & 0,75 & & $\mathrm{x}$ \\
\hline ALGODÃO DO PARÁ & HIBISCUS TILLACEUS L. & 5 & 1,88 & $\mathrm{x}$ & \\
\hline AZEITONA & SYZYGIUM JAMBOLANUM (LAM)DC. & 3 & 1,12 & $\mathrm{x}$ & \\
\hline CAJARANA & SPONDLAS CYTHEREA & 1 & 0,37 & & $\mathrm{x}$ \\
\hline CAJUEIRO & ANACARDIUM OCCIDENTALE & 5 & 1,88 & & $\mathrm{x}$ \\
\hline CARNAÚBA & COPERNICLA PRLNIFERA & 16 & 6,01 & & $\mathrm{x}$ \\
\hline CASTANHOLA & TERMINALLA CATAPPA L. & 5 & 1,88 & $\mathrm{x}$ & \\
\hline COQUEIRO & COCOS NUCIFERA L. & 83 & 31,2 & $\mathrm{x}$ & \\
\hline EUCALIPTO & EUCALYPTUS GLOBULUS & 7 & 2,63 & $\mathrm{x}$ & \\
\hline ESPIRRADEIRA & NERIUM OLEANDER L. & 1 & 0,37 & $\mathrm{x}$ & \\
\hline FLAMBOYANT & DELONLX REGLA (BOJER EX HOOK) RAF. & 3 & 1,12 & $\mathrm{x}$ & \\
\hline FIGO & OPUNTLA BRASILIENSIS & 2 & 0,75 & & $\mathrm{x}$ \\
\hline IMBURANA & BURSERA LEPTOPHLOEOS & 2 & 0,75 & & $\mathrm{x}$ \\
\hline JUAZEIRO & ZIZIPHUS JOAZEIRO MART. & 9 & 3,38 & & $\mathrm{x}$ \\
\hline JUCÁ OU PAU-FERRO & CAESALPINLA FERREA & 2 & 0,75 & & $\mathrm{x}$ \\
\hline LIMLÃO & CITRUS LIMON & 2 & 0,75 & $\mathrm{x}$ & \\
\hline LIRIO & SPATHIPHYLLUM WALLISII & 5 & 1,88 & $\mathrm{x}$ & \\
\hline LEUCENA & LEUCAENA LEUCOCEPHALA (LAM) DE WIT & 4 & 1,5 & $\mathrm{x}$ & \\
\hline MANQUEIRA & MANGIFERA INDICA L. & 11 & 4,13 & $\mathrm{x}$ & \\
\hline NIM & AZADIRACHTA INDICA A. JUSS. & 70 & 26,3 & $\mathrm{x}$ & \\
\hline OITICICA & IICANLA RIGIDA & 1 & 0,37 & & $\mathrm{x}$ \\
\hline PALMEIRA-IMPERIAL & ROYSTONEA OLERACEA & 5 & 1,88 & $\mathrm{x}$ & \\
\hline PATA OU MÃO DE VACA & BAUHINLA FORFICATA LINK & 1 & 0,37 & & $\mathrm{x}$ \\
\hline PAU-BRASII & CAESALPINLA ECHINATA & 2 & 0,75 & & $\mathrm{x}$ \\
\hline PINHEIRO & ARAUCARLA ANGUSTIFOLLA & 3 & 1,12 & & $\mathrm{x}$ \\
\hline QUIXABEIRA & SIDEROXYLON OBTUSIFOLIUM & 2 & 0,75 & & $\mathrm{x}$ \\
\hline TAMARINDO & TAMARINDUS INDICA L. & 11 & 4,13 & $\mathrm{x}$ & \\
\hline TANGERINEIRA & CITRUS RETICULATA & 1 & 0,37 & $\mathrm{x}$ & \\
\hline IPË COMUM & TABEBULA LONGIFLORA & 4 & 1,5 & & $\mathrm{x}$ \\
\hline TOTAL & 29 & 266 & 100 & 15 & 14 \\
\hline
\end{tabular}

A conservação da biodiversidade é um aspecto que vem sendo incorporado, cada vez com maior ênfase, como um objetivo importante do manejo florestal. Mesmo que o papel da biodiversidade na sustentabilidade da produção florestal não seja ainda muito claro, existe uma demanda da sociedade para assegurá-la, seja através de sistemas de certificação do bom manejo florestal, seja através de disposições legais e técnicas.

As árvores são consideradas essenciais em espaços urbanos, uma vez que estas influenciam na melhoria dos fatores climáticos, como um fator determinante sobre o microclima local (LOMBARDI; MORAIS, 2003; OLIVEIRA, 2011).

Outro aspecto importante a ser considerado é que estas estão diretamente relacionadas à conservação da diversidade da fauna local, servindo de refúgio para as espécies remanescentes das cidades (OLIVEIRA, 2011).

No entanto, um conceito importante a destacar é que a biodiversidade está normalmente associada ao equilíbrio dos ecossistemas, o que poderia sugerir que a biodiversidade poderia ser parte constituinte desta sustentabilidade.
Tal percentagem mostra que há a dominância da espécie do coqueiro (Cocos nucifera L.) Com percentagem de $31,2 \%$, no bairro de peixe-gordo em Icapuí, isso pode ser justificado pelo fato da região ser tipicamente de vegetação litorânea (figura 1).

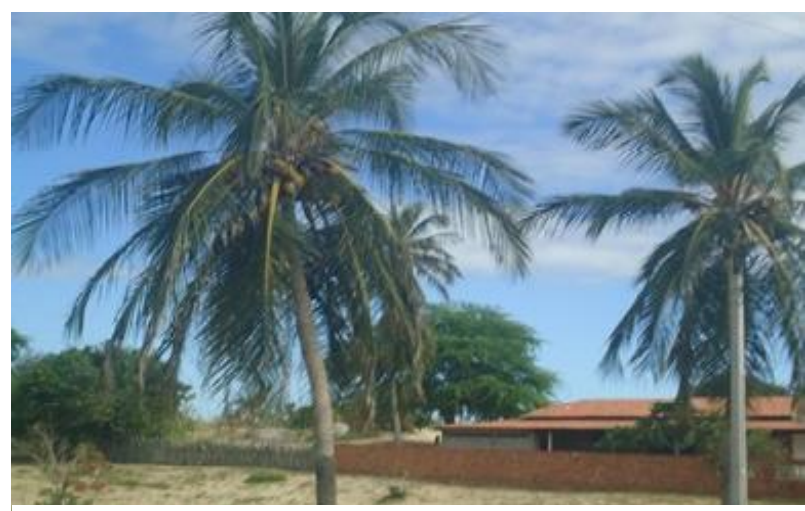

Figura1: Imagem do coqueiro (Cocos nucifera L.) A espécie mais encontrada no bairro peixe-gordo com cerca de 31,2. 
As árvores são consideradas essenciais em espaços urbanos, uma vez que estas influenciam na melhoria dos fatores climáticos, como um fator determinante sobre o microclima local (LOMBARDI; MORAIS, 2003; OLIVEIRA, 2011). Outro aspecto importante a ser considerado é que estas estão diretamente relacionadas à conservação da diversidade da fauna local, servindo de refúgio para as espécies remanescentes das cidades (OLIVEIRA, 2011).

Arborização em centros urbanos auxilia não somente na melhoria dos aspectos paisagísticos, mas também beneficia a população ao redor, por estar relacionada com a saúde física e mental (ROSSATTO et al., 2008); sendo considerada um importante elemento para salubridade ambiental (PINHEIRO, 2012). Entretanto, para que se tenha um ambiente ecologicamente equilibrado é necessária à realização de um planejamento prévio para a escolha de espécies adequadas para a arborização, dando-se prioridade a espécies nativas que sejam adaptadas ao clima da região e que desempenhem uma função ecológica importante para o bioma (BRUNET al., 2007; LOBODA; DE ANGELIS, 2009).

Segundo ROSSATO et al.,(2008) afirma que a dominância de uma espécie varia de acordo com a cidade e região do brasil. Em número de espécies as plantas exóticas são mais predominantes na cidade, com 15 indivíduos arbóreos representando $(51,7 \%)$ das espécies, e 14 indivíduos arbóreos nativos representando (48,3\%).

Em relação á origem das espécies, constatou-se que cerca de $51,7 \%$ das espécies arbóreas utilizadas na arborização do bairro peixe-gordo na cidade de Icapuí são consideradas exóticas. Em contrapartida 48,3\% são espécies arbóreas consideradas nativas. $\mathrm{O}$ plantio das plantas exóticas pela própria população é revelado pelo índice considerável representado no gráfico 1

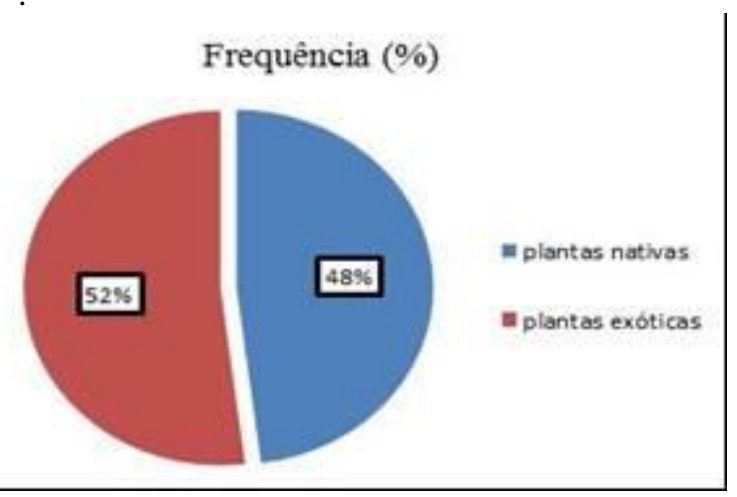

Gráfico1: Distribuição Dos Indivíduos Arbóreos Com Frequência Para Plantas Nativas E Exóticas No Bairro De Peixe-Gordo Na Cidade De Icapuí-Ce

As árvores são o mais perfeito tipo de cobertura vegetal para proteção do solo contra os efeitos danosos provocados pela erosão hídrica, uma vez que suas frondes anulam o impacto das gotas d'água contra o solo. Isto porque a água da chuva flui gradual e lentamente pela ramagem até o solo, neste se infiltrando e se depositando, contribuindo para o aumento das reservas hídricas subterrâneas.
O conhecimento da vegetação já existente é primordial para definir procedimentos, especialmente os relacionados às técnicas de manejo dessa vegetação e à seleção das espécies que serão introduzidas, considerando-se suas exigências naturais e o atendimento aos interesses dos usuários.

As árvores, em muitos casos, por serem redutos de espécies da fauna e da flora, inclusive daquelas ameaçadas de extinção, desempenham papel de suma relevância para a preservação destas espécies, ampliando sua importância ecológica. (figura 2).

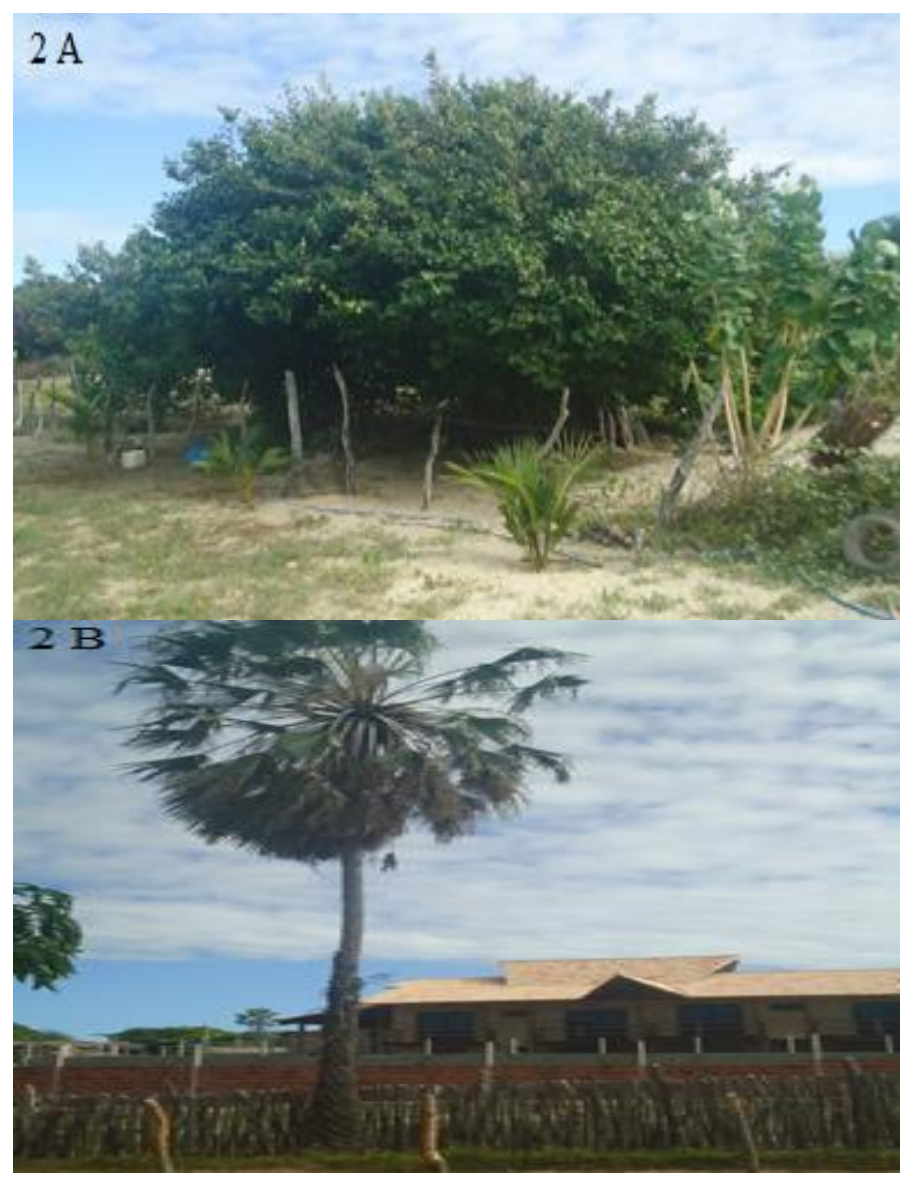

Figura 2: imagem do juazeiro (2A) e da carnaúba (2B) representando espécies nativas

Segundo LINDENMAIER et al., (2008) A Superultilização de espécies exóticas na arborização de Áreas verdes urbanas, pode ser atribuída em parte a um reflexo de tendências paisagísticas anteriores, pois, sob o ponto de vista estético, simplesmente e mais fácil encontrar espécies de grande beleza distribuídas por todo o mundo, do que somente me Um espaço geográfico ou formação vegetal restrita (figura 3).

Nas ruas e avenidas das cidades brasileiras, Predominam, hoje, árvores de espécies exóticas, ou seja, que não são espécies brasileiras, totalizando, aproximadamente, $80 \%$ dos exemplares (figura 4).

Figura 4: imagem do tamarindo, espécie exótica no bairro peixe-gordo na cidade de Icapuí-ce

Tendo em vista a necessidade de manutenção e conservação da diversidade das espécies da flora 
nacional, recomenda-se, sempre que possível, o emprego de plantas nativas da região, as quais têm a vantagem de estarem plenamente adaptadas às condições de solo e clima locais.

Embora a diversidade da flora brasileira seja a mais representativa de todo o planeta, há dificuldades de opção por determinadas espécies em razão da escassez de dados de pesquisa sobre o assunto. Apesar disso, deve-se indicar e adotar para plantio em passeios de vias públicas árvores com características favoráveis, com eficiência consagrada, e cujas qualidades e efeitos estejam comprovados pela prática.

No gráfico 2, abaixo, pode-se perceber que o número de ocorrência do coqueiro, Nim, carnaúba, mangueira, tamarindo e juazeiro estão em destaque em relação a todas as 29 espécies encontradas no levantamento. Como em muitos lugares o bairro peixe-gordo não possui planejamento de arborização, por isso, os plantios são considerados contínuos e aleatórios feitos pela própria população.

Figura 3: imagem do nim (3A) e da mangueira (3B) representando espécies exóticas
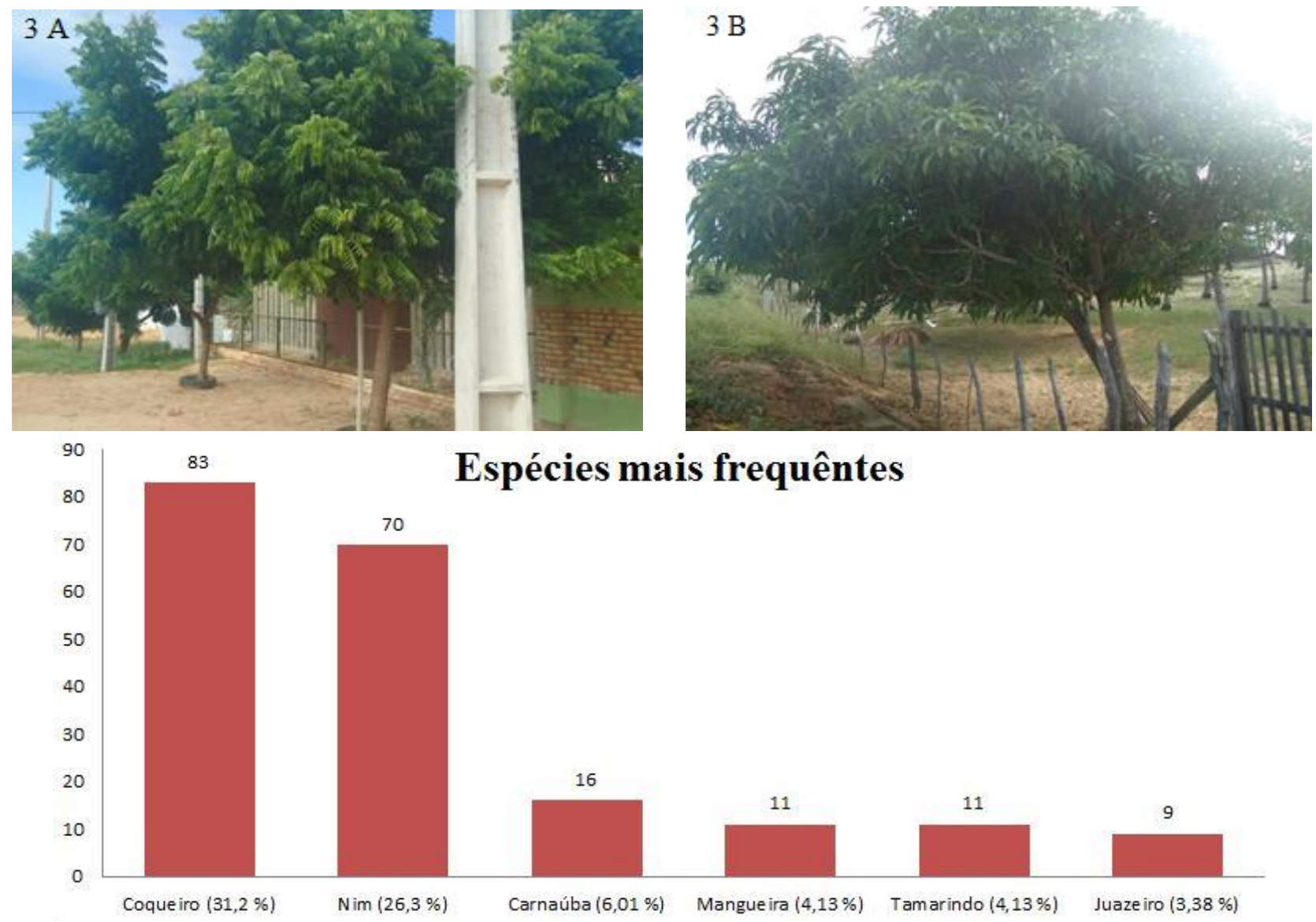

Gráfico 2: número de ocorrências das espécies mais frequêntes no bairro peixe-gordo em Icapuí-ce

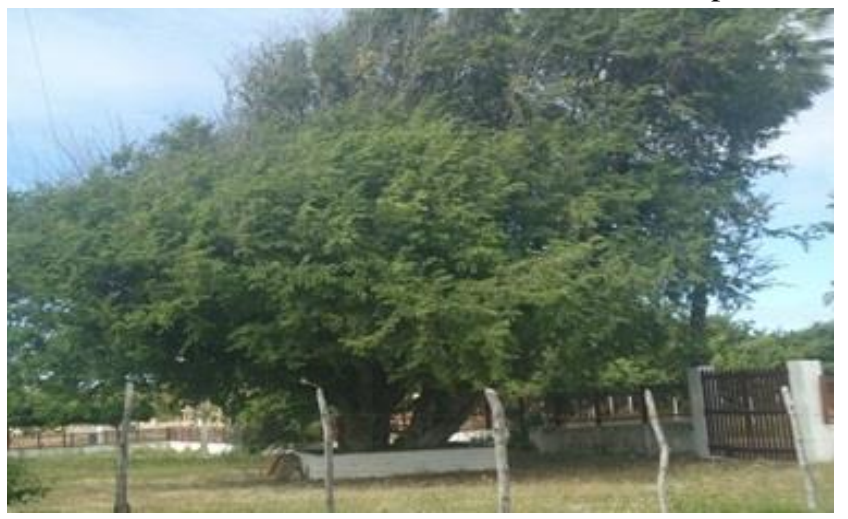

\section{CONCLUSÃO}

A quantidade de árvores nativa na atual situação da arborização urbana do bairro de peixe-gordo na cidade de Icapuí foi considerada razoável. A flora exótica presente na arborização do bairro peixe-gordo possui 15 espécies representando $(51,7 \%)$ das espécies e 14 espécies consideradas nativas $(48,3 \%)$. Dentre todas as 29 espécies, a mais frequente foi a do coqueiro (Cocos nucifera L.) Com uma percentagem de $31,2 \%$.

Foi observado que entre todas as 29 espécies arbóreas encontradas, 5 se destacaram em número de ocorrência: nim (Azadirachta indica a. Juss.) com percentual de 
26,3\%, carnaúba (Copernicia prunifera) com 6,01\%, mangueira (Mangifera indica L.) e tamarindo (Tamarindus indica L.) com $4,13 \%$ respectivamente, e o juazeiro (Ziziphus joazeiro mart.) com $3,38 \%$ de ocorrência.

\section{REFERÊNCIAS}

Estudo De Impacto Ambiental - Eia Para Atividade De Produção E Escoamento De Petróleo E Gás No Campo De Siri - Bacia Potiguar (PETROBRAS 2005).

Ceará/srh. Diagnósticogeoambiental e socioeconômico das áreas estuarinas e manguezais do rio jaguaribe, no estado do ceará/secretaria de recursos hídricos.fortaleza Ceará:srh/funceme/(SEMACE,2005).

SOUZA ,m.j.n, diagnóstico da zona costeira do estado do Ceará. Fortaleza-ce, 2003.

BONAMETTI, J. H. A ação do IPPUC na transformação da paisagem urbana de Curitiba a partir da área central. 2000. Dissertação(Mestrado em Arquitetura e Urbanismo, Área de Tecnologia do Ambiente Construído)-Escola de Engenharia de São Carlos, Universidade de São Paulo - EESC/USP, São Carlos.

LINDENMAIER, d. S.; santos, n. O. Arborização urbana das praças de caçhoeira do sul-rs-brasil: fitogeografia, diversidade e índice de áreas verdes. Instituto ançhietano de pesquisas, são leopoldo-rs, botânica n. 59, p. 307-320, 2008.
ROSSATO, d.r.; tsuboy, m.s.f.; frei, f. Arborização urbana na cidade de assis-sp: uma abordagem quantitativa. Revista sbau, piracicaba, v.3, n.3, p.1-16. 2008.

BATIONO, a. B.; yelemou, b.;ouedraog, s. J. Le neem (azadirachta indicaa. Juss.), une espèceexotiqueadoptée par lespaysansdu centre-ouestduburkinafaso. Bois etforêts des tropiques, n. 282, p. 5-10, 2004.

DRECHSEL, p.; zech, w. Foliar nutrient levels of broadleaved tropical trees: a tabular review. Plantandsoil, v. 131, p. 2946, 1991.

MARTINEZ, s. S. (ed.). O nim -azadirachta indica: natureza, usos múltiplos, produção. Londrina: iapar, 2002. $142 \mathrm{p}$.

NEVES, e. J. M. ;carpanezzi, a. A. Teores foliares de nutrientes em plantios de nim nos estados do paraná e são paulo. Colombo: embrapa florestas, 2006. (embrapa florestas. Comunicado técnico, 168).

NEVES, e. J. M.; carpanezzi, a. A. (ed.). A cultura do nim (azadirachta indica a. Juss.). Brasília, df: embrapa informação tecnológica, 2008. (coleção plantar). No prelo.

TEIXEIRA, i. F. Análise quantitaticada arborização de ruas do conjunto habitacional tancredo neves, santa maria-rs. Revista ciência florestal.v.9, n.2, p. 9-21, 1999. 